\title{
A Recall Reaction and Call for Action
}

\author{
Seppo W. Langer \\ Dept. of Oncology 5073, Copenhagen University Hospital, Rigshospitalet, Copenhagen, Denmark
}

Radiation recall reactions are inflammatory reactions that occur in previously irradiated areas, and are precipitated by a recall-triggering drug. The drug is often, but not always, an antineoplastic agent. The reaction has been known for more than fifty years, and may occur in any localisation of the body. In fact, radiation recall reactions have been described in the mucous membranes, the nervous tissues, respiratory and gastrointestinal tract etc. Radiation recall dermatitis refers to a recall reaction in the skin. The incidence is low, and our knowledge of the features of the recall reactions is therefore mainly based on clinical experience and published case reports; the latter are summarized and discussed in several recent reviews [1-4].

Recall reactions often occur between a week and two years after the radiation therapy. The list of triggering drugs is long; however antineoplastic agents, such as the anthracyclines, actinomycin D, metotrexate, and the taxanes, are associated with the highest reported incidences. Recall dermatitis presents as erythema, vesicles and desquamation of the affected skin areas. The affection is nearly always confined to the radiation port. The onset of the reaction may vary from minutes to several weeks after introduction to the trigger drug, and the skin changes generally resolve gradually during the weeks after discontinuation of the inciting agent. There seems to be no direct association between acute radiation damage, late radiation sequelae, and radiation recall dermatitis. The radiation doses in reported cases range from 10 to $81 \mathrm{~Gy}$. The exact mechanism of action is not known.

Possible aetiological factors are proposed to be a combination of radiation induced stem cell dysfunction, vascular changes and local immune changes, which change the local threshold for an idiosyncratic hypersensitivity reaction to the trigger drug.

Treatment options include discontinuation of the trigger drug, and local and/or systemic steroids. Symptoms may be eased by antihistamines. Re-challenge with the triggering agent has been carried out without the precipitation of a new reaction. Hence, permanent discontinuation of the drug may actually not be mandatory. No uniform treatment guideline has yet gained universal acceptance.

In this issue of ONKologie, Zhu et al. [5] describe a patient who developed radiation recall dermatitis during treatment with cisplatin and vinorelbine 1 month after the completion of radiation therapy for lung cancer. The skin reaction was treated with antihistamine and local steroid and disappeared after 3 weeks. The authors suggest that the case serves as a warning of potential risk of radiation recall reactions during treatment with cisplatin and vinorelbine. The drug combination is often used in patients that at some point during their cancer treatment may also be treated with radiotherapy, before as well as concomitantly and after chemotherapy. Therefore a general warning is not necessary. However, the case story by Zhu et al. may actually serve as a reminder to the oncology community to increase the awareness of a number of treatment related dermatoses. Such awareness may lead to improved clinical outcome and thus better patient care.

For example, accidental extravasation of chemotherapy is a potentially devastating side effect of treatment with the classic antineoplastic drugs, especially the vesicant drugs, e.g. the anthracyclines and the vinca alkaloids. Numerous published case reports of extravasation injuries resulted in focused experimental search for potential antidotes which then further resulted in the introduction of effective treatments. Recently, revised international treatment guidelines were published $[6,7]$.

The introduction of several new targeted biological therapies has resulted in novel toxicity profiles, including dose limiting cutaneous toxicity. The marked skin toxicity associated with inhibition of EGFR, both by the small molecule tyrosine kinase inhibitors and several monoclonal antibodies, has attracted considerable attention [8]. The clinical importance of the cutaneous side effects of these new agents should not be underestimated.

\section{KARGER}

Fax +497614520714

Information@Karger.de

www.karger.com (c) 2010 S. Karger GmbH, Freiburg

Accessible online at:

www.karger.com/onk 
At present much of the dermatological care of patients with toxicity of the skin and appendages focuses on damage control. Hence, there seem to be considerable dermato-oncological challenges ahead with respect to uncovering, analysing, preventing, and treating dermatoses related to cancer treatment.

\section{References}

1 Azria D, Magné N, Zouhair A, Castadot P, Culine S, Ychou M, Stupp R, Van Houtte P, Dubois JB, Ozsahin M: Radiation recall: a well recognized but neglected phenomenon. Cancer Treat Rev 2005;31:555-570.

2 Camidge R, Price A: Characterizing the phenomenon of radiation recall dermatitis. Radiother Oncol 2001;59:237-245.

3 Caloglu M, Yurut-Caloglu V, Cosar-Alas R, Saynak M, Karagol H, Uzal C: An ambiguous phenomenon of radiation and drugs: recall reactions. Onkologie 2007;30:209-214.

4 Hird AE, Wilson J, Symons S, Sinclair E, Davis M, Chow E: Radiation recall dermatitis: Case report and review of the literature. Curr Oncol 2008;15:53-62.

5 Zhu ZF, Fan M, Fu XL: Radiation recall with vinorelbine and cisplatin. Onkologie 2010;33: DOI: $10.1159 / 000277672$

-6 Wengström Y, Margulies A; European Oncology Nursing Society Task Force: European Oncology Nursing Society extravasation guidelines. Eur J Oncol Nurs 2008;12:357-361.

7 Schulmeister L: Vesicant chemotherapy extravasation antidotes and treatments. Clin J Oncol Nurs 2009;13:395-398.

8 Agha R, Kinahan K, Bennett CL, Lacouture ME: Dermatologic challenges in cancer patients and survivors. Oncology 2007;21:1462-1472. 